\title{
Long-term efficacy of microbiology-driven periodontal laser-assisted therapy
}

\author{
F. S. Martelli ${ }^{1}$ E. Fanti ${ }^{2}$ C C. Rosati ${ }^{2}$ - M. Martelli $^{1} \cdot$ G. Bacci $^{3}$ • \\ M. L. Martelli ${ }^{2}$ E. Medico ${ }^{4}$
}

Received: 26 August 2015 / Accepted: 10 December 2015 / Published online: 6 January 2016

(C) Springer-Verlag Berlin Heidelberg 2015

\begin{abstract}
Periodontitis represents a highly prevalent health problem, causing severe functional impairment, reduced quality of life and increased risk of systemic disorders, including respiratory, cardiovascular and osteoarticular diseases, diabetes and fertility problems. It is a typical example of a multifactorial disease, where a polymicrobial infection inducing chronic inflammation of periodontal tissues is favoured by environmental factors, life style and genetic background. Since periodontal pathogens can colonise poorly vascularised niches, antiseptics and antibiotics are typically associated with local treatments to manage the defects, with unstable outcomes especially in early-onset cases. Here, the results of a retrospective study are reported, evaluating the efficacy of a protocol (Periodontal Biological Laser-Assisted Therapy, Perioblast ${ }^{\mathrm{TM}}$ ) by which microbial profiling of periodontal pockets is used to determine the extent and duration of local neodymium-doped yttrium aluminium garnet (Nd:YAG) laser irradiation plus conventional treatment. The protocol was applied multicentrically on 2683 patients, and found to produce a significant and enduring improvement of all clinical
\end{abstract}

Electronic supplementary material The online version of this article (doi:10.1007/s10096-015-2555-y) contains supplementary material, which is available to authorized users.

F. S. Martelli

info@microdentistry.net

1 Microdentistry, Via dell'Ariento 4, 50123 Florence, Italy

2 Biomolecular Diagnostic srl, Via Nicola Porpora 5, 50144 Florence, Italy

3 Department of Biology, University of Florence, Via Madonna del Piano 6, 50019 Sesto Fiorentino, Firenze, Italy

4 Department of Oncology, University of Torino, Regione Gonzole 10, 10043 Orbassano, Torino, Italy and bacteriological parameters, even in aggressive cases. Microbiome sequencing of selected pockets revealed major population shifts after treatment, as well as strains potentially associated with periodontitis in the absence of known pathogens. This study, conducted for the first time on such a large series, clearly demonstrates long-term efficacy of microbiology-driven non-invasive treatment of periodontal disease.

Keywords Periodontitis $\cdot$ Periodontal therapy $\cdot$ Bacterial infection $\cdot$ Dental practice $\cdot \mathrm{Nd}$ : YAG laser $\cdot$ Perioblast $\mathrm{T}^{\mathrm{TM}}$

\section{Introduction}

Periodontitis is a complex inflammatory multifactorial disease, caused by the disruption of normal homeostatic processes by numerous bacterial species present in subgingival dental plaque [1]. It affects the support structures of the teeth and, if untreated, may result in pain, impaired mastication and tooth loss [2]. Microcolonies of oral pathogens attach to the tooth surface, infiltrate periodontal tissues and organise a subgingival plaque biofilm. Although the microbial infection is the main aetiological factor, the pathogenesis of periodontitis is a complex interaction between the microbiome, host tissues and individual immune response [3], further modified by environmental factors, age and smoking [4]. In fact, periodontitis occurs when pathogenic bacterial species begin to dominate, overruling host defence and triggering an enhanced immune response, which may persist, leading to dysregulated and non-effective chronic inflammation [1]. Moreover, there is increasing evidence of a strong correlation between the presence of pathogenic bacteria in periodontal lesions and several systemic diseases. In particular, bacteraemia and systemic endotoxin release can lead to the onset and progression of respiratory and cardiovascular diseases, rheumatoid arthritis and diabetes mellitus [5]. 
Furthermore, oral bacteria can bypass the placenta filter, with a consequent increase of mediators responsible for infertility, preterm births and underweight newborns [6-8]. Traditionally, periodontitis is considered as a chronic disease, easy to relapse and without an effective therapy in the aggressive cases, that represent $10 \%$ of the total $[9,10]$. Treatment procedures involve scaling and root planing (SRP), local antiseptic and systemic antibiotic administration, periodontal surgery and, ultimately, extraction of compromised teeth. Indeed, post-therapy microbial rebound has been demonstrated, as periodontal pockets may be re-colonised by putative periodontal pathogens already one week after mechanical debridement $[11,12]$.

An increased and lasting decontamination of the periodontal pockets and of the oral ecosystem, together with radiologically detectable bone mineralisation and regeneration, can be achieved through the use of high-powered neodymium-doped yttrium aluminium garnet (Nd:YAG) laser [13]. The antimicrobial properties of laser allow the deep and lasting decontamination from pathogens and the inactivation of bacterial endotoxins in periodontal pockets, as well as in root canals and around implants [14]. Nd:YAG laser overcomes the resistance of subgingival biofilm communities to antibiotics traditionally used in dental practice and it exerts an antiseptic action also in tissues poorly or not vascularised, like bone or dentin, unreachable by drugs [15]. Moreover, laser irradiation promotes fibroblasts and osteoblasts activity, likely through the activation of BMP-2-related signalling pathways, resulting in an increase of collagen production during the periodontal healing phase [16-18]. All the above studies, however, have been conducted on an extremely limited number of cases and require more extensive validation. The primary aim of this retrospective study, performed for the first time on 2683 patients and on 13,550 periodontal pockets, with a long-term follow-up (24 months), is to provide a statistically robust evaluation of the clinical and microbiological efficacy of nonsurgical laser periodontal treatment in conjunction with SRP. A secondary aim of the study was to reach, through metagenomics analysis, a deeper characterisation of bacterial species involved in periodontitis and to highlight novel candidate periodontal pathogens.

\section{Methods}

Detailed information is described in the Supplementary material.

\section{Generation of the dataset}

A series of 2683 periodontal patients, treated by various clinicians in six different clinics, has been retrospectively assessed. Patients were anonymised and informed consent was obtained from all subjects.

\section{Laser irradiation of periodontal pockets}

The standard Nd:YAG laser setting parameters were: frequency $20 \mathrm{~Hz}, 2$ Watt, 100 mJoule, 50/100 $\mu$ s pulse duration. Each patient underwent at least four sessions of laser treatment until a stable microbiological shift occurred.

\section{Sample collection}

Sampling was carried out following the procedures reported in the BPA kit (Bacterial Periodontal Assessment, Biomolecular Diagnostic, Firenze, Italy).

\section{Assessment of periodontopathic bacteria}

Plaque samples were sent to Biomolecular Diagnostic (Firenze, Italy). The DNA extraction was performed using QIAsymphony (QIAGEN), according to the manufacturer's protocol. Real-time polymerase chain reaction (PCR) with SYBR Green assays were carried out using Rotor-Gene Q (QIAGEN) apparatus to quantify the most important periodontopathogens, including Porphyromonas gingivalis, Treponema denticola and Tannerella forsythia, Peptostreptococcus micros, Fusobacterium nucleatum ssp. polymorphum, Prevotella intermedia, Campylobacter rectus, Eikenella corrodens and Aggregatibacter actinomycetemcomitans.

\section{Statistics}

All statistical and distribution analyses were carried out with Excel (Microsoft). Distributions of the various clinical (PPD, BOP\%, PUS\%) and bacteriological (TOT BAC, RED, ORANGE, GREEN) variables at diagnosis and at different follow-up times were compared by Student's $t$-test.

\section{S rRNA metagenomics of the microbial community and NGS raw data processing of samples}

The microbial DNA of patients was quantified by the BioPhotometer (Eppendorf) and 16S rRNA sequencing was performed using HiSeq 2000 (Illumina). Patterns of microbial community structure were analysed from the samples collected. The V4 region of the 16S rRNA gene was PCR amplified by using the NEXTflex ${ }^{\mathrm{TM}}$ 16S V4 Amplicon-Seq Kit (Bioo Scientific Corporation, Austin, TX, USA) following the manufacturer's protocol, and the resulting amplicons ( $\sim 250 \mathrm{bp}$ long) were cleaned, quantified and sequenced on the Illumina MiSeq platform using paired 150-bp reads. Sequences analysis was performed by using the Metagenomics workflow of MiSeq Reporter v2.3 (Illumina). 


\section{Study approval}

Within the manuscript, there are no experiments requiring appropriate institutional review board(s) approval.

\section{Results}

\section{Clinical response: site-level analysis}

This retrospective study has been conducted on a consecutive series of 2683 periodontal patients (Table 1), profiled and treated according to a protocol (Periodontal Biological Laser-Assisted Therapy, Perioblast ${ }^{\mathrm{TM}}$ ) based on Nd:YAG laser irradiation of periodontal pockets guided by qualitative and quantitative assessment of bacterial populations in the pockets, as illustrated in Supplementary Figure 1. Further details are provided in the Supplementary material. Periodontal probing depth (PPD), bleeding on probing (BOP) and suppuration (PUS) were assessed before and after treatment in 13, 550 pockets, for up to $12-24$ months (Table 2 ). The mean pocket depth was reduced from $6.25 \mathrm{~mm}$ before treatment to $4.24 \mathrm{~mm}$ after $1-4$ months, $4.04 \mathrm{~mm}$ after $4-12$ months and $3.80 \mathrm{~mm}$ after 12-24 months (Fig. 1a). Similarly, BOPpositive sites decreased from $91 \%$ to around $50 \%$ (Fig. 1b) and PUS-positive sites decreased from $46 \%$ to around $2 \%$, over the same time intervals (Fig. 1c). No differences were observed when considering gender. PPD reduction was also analysed in subgroups with different ranges of PPD at diagnosis: 4-6 mm, 7-9 $\mathrm{mm}$ and $>9 \mathrm{~mm}$. Reduction was significant at all follow-up times, independently of the extent of PPD at diagnosis (Fig. 1d), and completely overlapping also in young patients $(<35$ years old; $n=1380$; Supplementary material and Supplementary Figure 2).

\section{Bacteriological response}

The microbiological status of periodontal pockets was assessed at diagnosis and after 1-4 months, 4-12 months and 12-24 months following treatment (see the Supplementary material). The average total bacterial load was drastically reduced, progressively decreasing from 53 to 16 million (T-test $p$-values: $<10^{-7}$ at $1-4$ months, $<10^{-12}$ at $4-12$ months and $<10^{-16}$ at $12-24$ months). Reduction of

Table 1 Demographic characteristics of the 2683 periodontal patients

\begin{tabular}{ll}
\hline Age, years (mean \pm standard deviation) & $49.72 \pm 12.1$ \\
$<35$ years old & $265(9.9 \%)$ \\
Females & $1561(58.2 \%)$ \\
Smokers & $940(35 \%)$ \\
Caucasian ethnicity & $2683(100 \%)$ \\
\hline
\end{tabular}

known pathogens was even more marked, from 5.3 million to 610,000 on average (T-test $p$-values: $<10^{-8}$ at $1-4$ months, $<10^{-7}$ at $4-12$ months and $12-24$ months; Fig. 2a). Within pathogens, the distribution of the red, orange and green Socransky's complexes [19] was then considered, given their involvement in different phases of the periodontal disease. In particular, prevalence of the red complex ( $P$. gingivalis, $A$. actinomycetemcomitans, $T$. denticola, $T$. forsythia and P. micros) is associated with advanced periodontitis and PPD of $6 \mathrm{~mm}$ or deeper, while prevalence of the orange complex (F. nucleatum ssp., P. intermedia and C. rectus) is associated with either the progression of the disease from mild to advanced or to its initial regression. Prevalence of the green complex (E. corrodens) is typically associated with the final phase of biological healing [20]. Changes in the distributions of the red, orange and green complexes are shown in Fig. $2 b$. The average load of all three complexes decreased drastically, but the decrease of the red complex was the most striking, already significant at 1-4 months and lasting until 12-24 months. In accordance with clinical observations, no significant differences in the response were observed when considering gender (see the Supplementary material). In the group of young patients, the average total bacteria load progressively decreased from 61 to 17 million and the known pathogens fraction decreased from 3.7 million to 390,000 (Fig. 2c). The lower amount and the distribution of the pathogens among the three complexes at baseline and after treatment are illustrated in Fig. 2d.

\section{Metagenomics}

In a small fraction of cases (approximately $10 \%$ ), known periodontal pathogens were not detected or their levels were not high enough to explain the clinical signs and severity of periodontitis. To explore the presence of bacterial signatures related to periodontal disease, next-generation sequencing (NGS)-based microbiome analysis was carried out on the periodontal pockets of 20 cases, subdivided into three groups as follows. The first group ('Pretreatment') included six periodontal patients affected by aggressive periodontitis with the presence of known pathogens, before any treatment. The second group ('Treated') was composed of samples taken from the same patients described above, but after successful Perioblast ${ }^{\mathrm{TM}}$ treatment. Finally, the third group ('False Negatives') included eight samples from patients clinically affected by advanced periodontal disease (PPD $>6 \mathrm{~mm}$, BOP and/or PUS), for which the results of microbiological tests revealed a very low percentage of known pathogens without the presence of periodontal species belonging to the red complex, also after repetition of the entire procedure of sampling and PCR analysis. NGS analysis was performed on the 16S rRNA gene from DNA extracted and amplified from 
Table 2 Summary of the microbiological and clinical parameters before and after treatment

\begin{tabular}{lllll}
\hline Variable & Baseline & $1-4$ months & $4-12$ months & 12-24 months \\
\hline N. of analysed patients & 2683 & 2090 & 634 & 684 \\
Average total bacteria & $53,760,815$ & $29,418,693$ & $20,677,076$ & $16,013,911$ \\
Red complex & $3,171,051(58.9 \%)$ & $93,122(14.5 \%)$ & $89,690(18.3 \%)$ & $173,404(28.3 \%)$ \\
Orange complex & $1,758,019(32.7 \%)$ & $237,180(36.8 \%)$ & $243,979(49.7 \%)$ & $389,495(63.4 \%)$ \\
Green complex & $424,099(7.9 \%)$ & $286,521(44.5 \%)$ & $129,389(26.3 \%)$ & $105,603(17.2 \%)$ \\
Average total pathogens & $5,379,946(10 \%)$ & $643,851(2.2 \%)$ & $490,960(2.4 \%)$ & $614,128(3.8 \%)$ \\
N. of analysed pockets & 13,590 & 9977 & 3060 & 3246 \\
Average PPD & $6.25 \mathrm{~mm}$ & $4.24 \mathrm{~mm}$ & $4.04 \mathrm{~mm}$ & $3.80 \mathrm{~mm}$ \\
BOP $^{\mathrm{b}}$ & $91 \%$ & $52 \%$ & $49 \%$ & $53 \%$ \\
PUS $^{\mathrm{c}}$ & $46 \%$ & $1 \%$ & $1 \%$ & $2 \%$ \\
\hline
\end{tabular}

${ }^{\text {a }} P P D$ periodontal probing depth

${ }^{\mathrm{b}} B O P$ bleeding on probing

${ }^{\mathrm{c}}$ PUS suppuration subgingival plaque samples as described (details in the Supplementary material).

First of all, samples were analysed by means of rarefaction curves [21]. All curves reached an asymptote, showing that the bacterial diversity was adequately estimated in all samples (Supplementary Figure 3). Taxonomic assignments obtained through NGS analysis were tabulated at the phylum level and used to generate the barplots reported in Fig. 3a, displaying the relative abundance of the phyla detected in each sample. Clearly, cases belonging to the same subgroup had a similar community structure, with major differences between pretreatment samples and the remaining two subgroups. Particularly, the elimination of the spirochaetes (orange bars) in the Treated samples was evident, along with their extremely low levels in the False Negatives group. Conversely, proteobacteria were prevalent in both the Treated and False Negatives groups. Accordingly, ANOSIM [22] and CCA [23] analyses confirmed that samples of the Pretreatment group tend to form a completely separate cluster, while clusters formed by the Treated and False Negatives groups display some degree of overlap (Supplementary Figure 4). However, significant ANOSIM $p$-values (less than 0.01 ) showed that bacterial communities associated with different states of periodontal disease have a distinct taxonomic structure at the phylum level.
Fig. 1 Clinical response: sitelevel analysis. a Average periodontal probing depth (PPD) reduction on 13,550 pockets with follow-up at different times. b Average bleeding on probing (BOP) reduction and $\mathbf{c}$ average suppuration (PUS) reduction on the same pockets. $\mathbf{d}$ Reduction of different PPD ranges at different times in the whole population
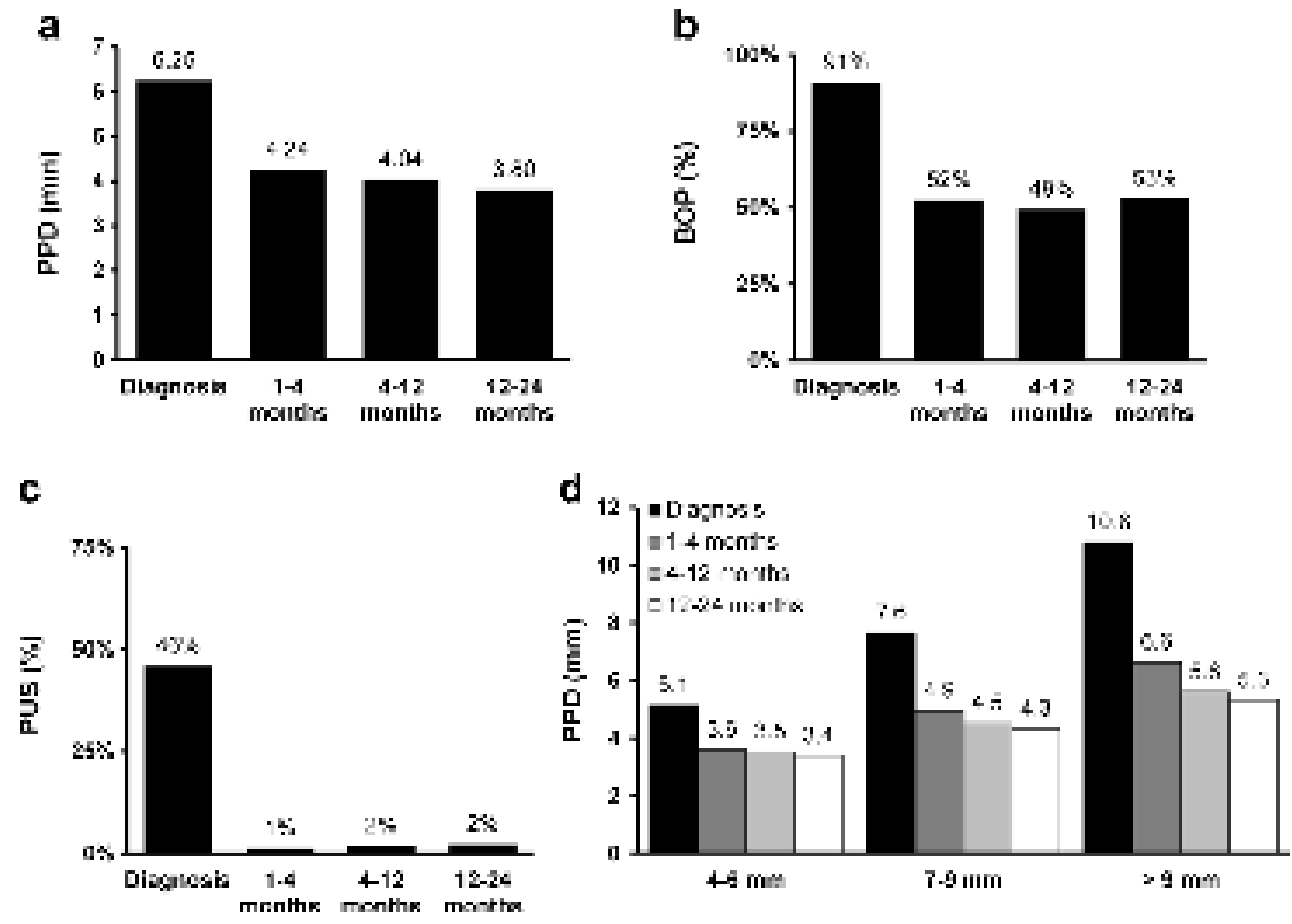

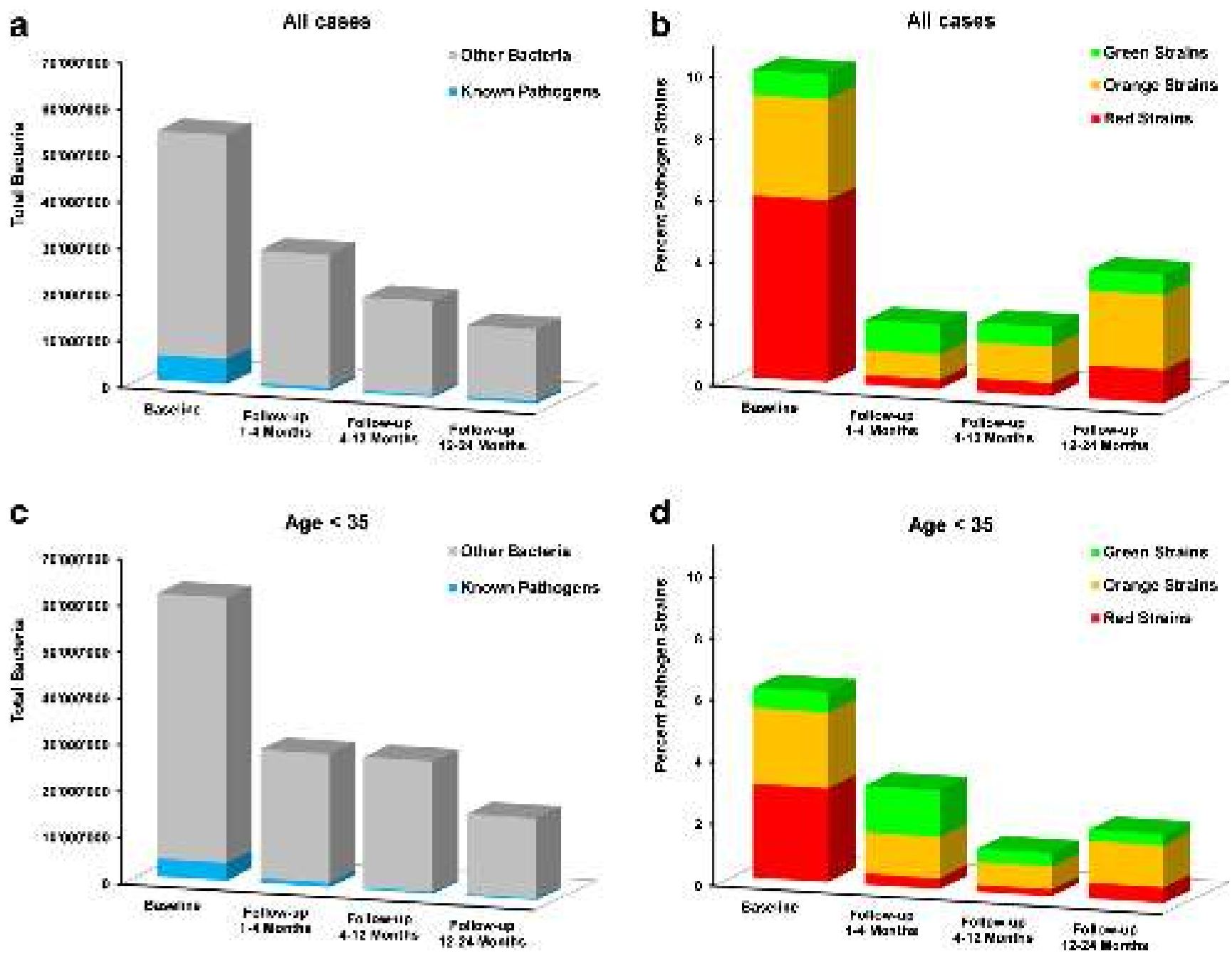

Fig. 2 Bacteriological response. a Average percentage of pathogens among total bacteria in 2683 patients with follow-up at 1-4 months and 12-24 months. b Distribution of red, orange and green complexes among pathogens in the same patients at the same times. $\mathbf{c}$ Average percentage of

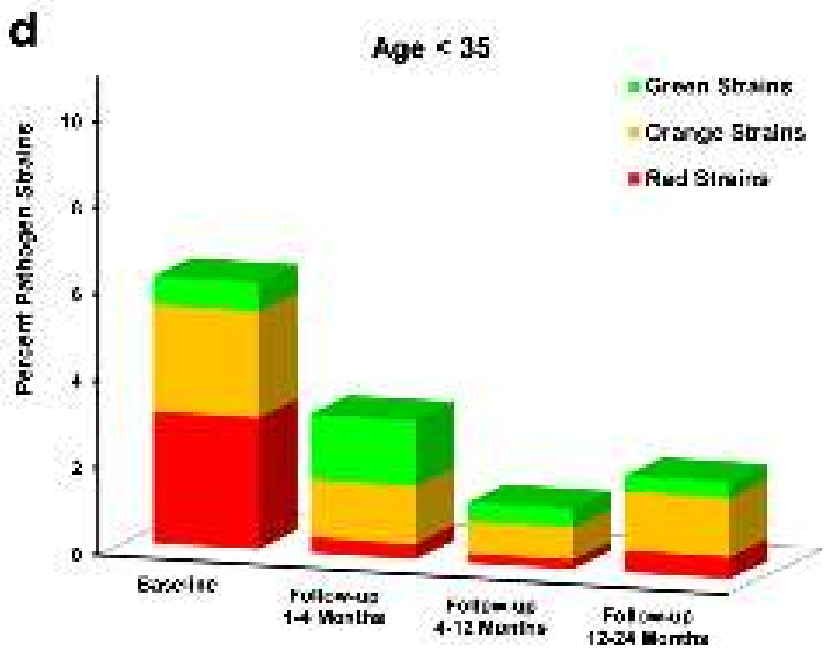

pathogens among total bacteria in 265 patients aged $<35$ years. d Distribution of red, orange and green complexes among pathogens in patients aged $<35$ years at the same times

To better characterise the bacterial species involved in periodontal disease, NGS data were analysed at the operational taxonomic unit (OTU) level. In 2009, Colombo and colleagues [24] described a bacterial signature distinguishing patients with treatment-refractory periodontitis from good responders and healthy cases. The signature included known pathogens, such as some red complex bacteria and C. gracilis, and 'unusual' species, including TM7 spp., Bacteroidetes sp., Veillonella atypica, Desulfobulbus sp., Brevundimonas diminuta, Gemella sanguinis, Mogibacterium tidmidum, Mycoplasma salivarium, Anaeroglobus geminatus, Megasphaera micronuciformis, Streptococcus parasanguinis, Pasteurella stomatis, F. naviforme and T. medium. It was, therefore, verified how these species are represented in our NGS dataset. As shown in Fig. 3b, red complex bacteria ( $T$. denticola, T. forsythia and P. gingivalis) were highly abundant in all Pretreatment samples and were almost ablated in

Treated cases. As expected, they were barely detectable in the False Negatives group. Interestingly, $C$. gracilis displayed a similar pattern of depletion upon treatment, but was also well represented in some of the samples of the False Negatives group (Fig. 3c). Among the unusual species, representation of Filifactor alocis, Bacteroidetes sp. and Desulfobulbus sp. was substantially overlapping with that of the red complex (Supplementary Figure 5), while TM7 and Dialister invisus were reduced by treatment but also clearly detectable in a fraction of the cases in the False Negatives group (Fig. 3d).

To identify new candidate pathogens in the False Negatives group, analysis of the similarity percentage (SIMPER analysis) [22] was carried out, comparing each group of samples with the others, to highlight OTUs with differential representation. The analysis revealed very clear differences between Pretreatment samples and either the Treated or False Negatives groups, and lower divergence between samples in the 
a
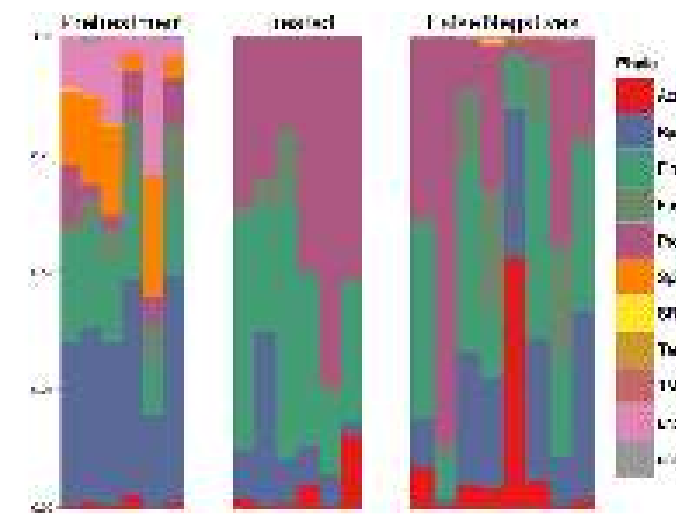

b
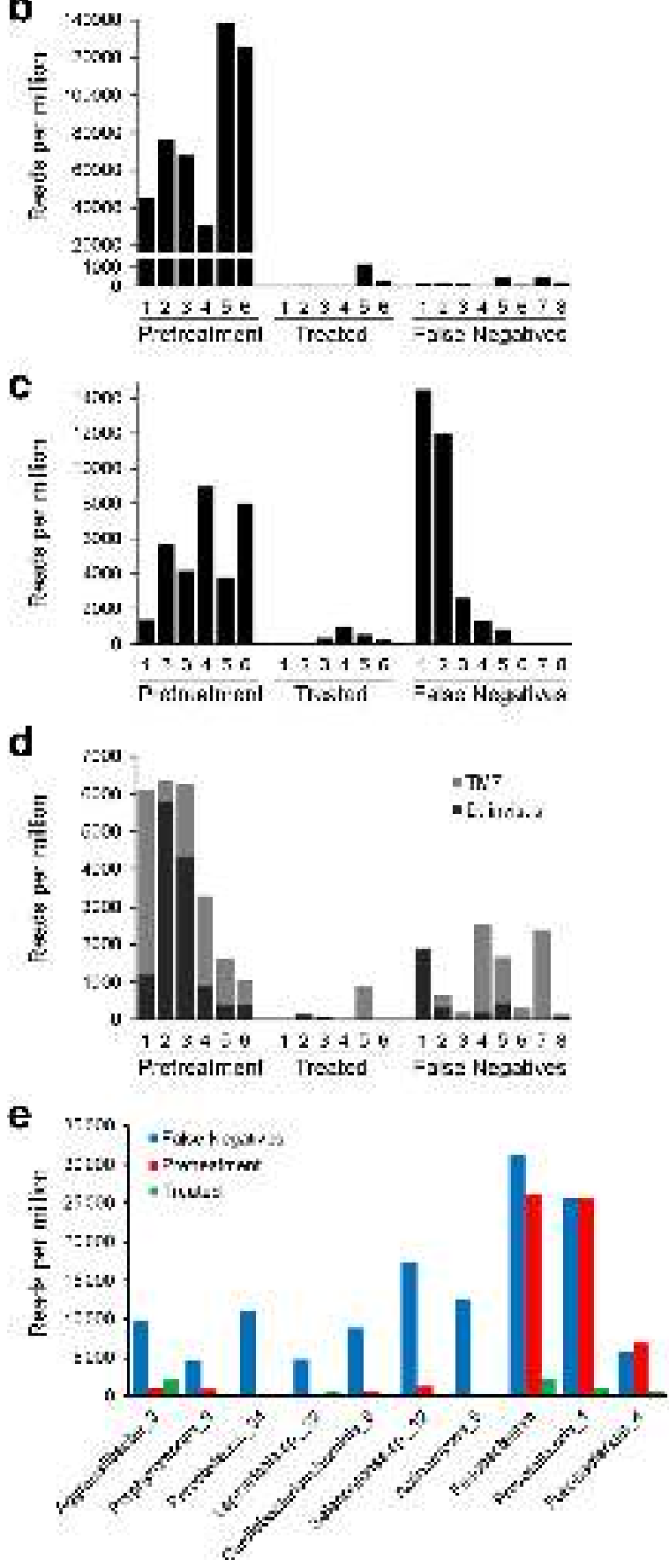

Fig. 3 Metagenomics. a Relative abundances of bacterial phyla detected in all samples. The colour patterns refer to nine distinct bacterial phyla and two unclassified ones. Samples were divided into three separated panels according to their group of patients, reporting the group name at the top of the panels. b Next-generation sequencing (NGS)-based calculation of the abundance of red complex bacteria (expressed as the sum of the reads per million of Treponema denticola, Tannerella forsythia and Porphyromonas gingivalis) in 20 samples, subdivided into Pretreatment, Treated and False Negatives groups, as indicated. The same abundance representation is displayed in $\mathbf{c}$ for Campylobacter gracilis and $\mathbf{d}$ for TM7 and Dialister invisus, all displaying a marked reduction in Treated cases, but also well detected in some of the False Negatives group. e Abundance, in terms of reads per million, of ten operational taxonomic units (OTUs) strongly associated with the False Negatives group. Three of them are also abundant in Pretreatment samples

Treated and False Negatives groups (Supplementary Figure 6). Starting from the 57 OTUs that, according to the SIMPER analysis, distinguish samples in the False Negatives group from those in the Treated group, we identified seven OTUs (Aggregatibacter 3, Porphyromonas 9, Prevotella sp._34, Leptotrichia sp._22, Cardiobacterium hominis 6, Selenomonas sp. 12, Actinomyces 5) specifically high in the False Negatives group and three that were also high in the Pretreatment samples (Fig. 3e).

\section{Discussion}

The present multicentric retrospective study demonstrates that microbiologically guided $\mathrm{Nd}$ :YAG laser irradiation of periodontal pockets, in conjunction with SRP, is an effective treatment for advanced periodontitis. The results of the present study are comparable to those described by some authors $[25,26]$, but better if compared to those reported by literature meta-analysis studies [27, 28]. Reduction of PPD, BOP and PUS in response to the treatment protocol was proven to occur early and last for 24 at least months. A clear tendency for continuous parameter improvement was observed, which reflects long-term treatment-induced normalisation of the periodontal microenvironment, favouring further regression of the lesions.

Many research groups have examined microbiological shifts in periodontal pockets after applying conventional non-surgical periodontal therapy to patients (including SRP alone or in combination with antibiotics and antiseptics) [29-37]. In comparison with our study, smaller samples of patients were used in these studies. Furthermore, periodontal pockets were re-colonised by measurable amounts of periodontal pathogens as early as 3 months after periodontal therapy. Although supplemental administration of antibiotics and antiseptics was more beneficial than SRP alone in general, follow-up for these studies lasted no longer than 12 months from the initial therapy. That happened because, by that time, 
periodontal pathogens levels had increased to the initial levels before treatment.

The fact that similar results have also been obtained in young patients is remarkable since, according to the current literature, periodontitis in young patients is considered aggressive, refractory or shortly responsive to treatments, with a quick progression of the tissue damage $[9,10]$. Considering the microbiological status of periodontal pockets, the average total bacterial load was drastically reduced at all follow-up times, with reduction of known pathogens being even more evident. These results reflect both improved short-term control compared to the current literature and unprecedented longterm control. In particular, among the three Socransky's complexes [19], the reduction in the red complex was the most notable, which is particularly relevant, given its association with advanced phases of periodontitis.

Interestingly, the $<35$ years old group was characterised by a significantly lower pathogen load at baseline, despite equally severe clinical parameters of disease. This suggests a role for individual genetic susceptibility, allowing a lower pathogen load to promote early onset of aggressive periodontitis. Also, the discrepancy between low pathogen load and clinical severity could be due to the presence of bacteria not currently recognised as periodontal pathogens. In this respect, laser treatment could provide substantial therapeutic benefits when added to conventional treatment. Indeed, the results obtained in this group of patients, similar to those obtained in the whole population, confirm also from the microbiological point of view the enduring efficacy of the laser therapy protocol, even in aggressive, early-onset periodontal disease. Studies investigating the microbiological shifts in periodontal pockets of patients with aggressive periodontitis after conventional treatment showed similar limitations to the respective studies that included patients with chronic periodontitis referred above, compared to our study (small sample, re-colonisation of periodontal pockets by periodontal pathogens after 3 months, follow-up no longer than 6 months) [38, 39].

In about $10 \%$ of cases, patients negative at diagnosis for known pathogens, in particular for the Socransky red complex, are clinically affected by advanced periodontitis. Therefore, NGS analysis of the microbiome was performed on periodontal pockets from eight such cases ("False Negatives"), from six cases affected by advanced periodontitis with high load of known pathogens at diagnosis ("Pretreatment") and from the same six patients after successful Perioblast ${ }^{\mathrm{TM}}$ treatment ("Treated"). Phylum level analysis confirmed that cases belonging to the same subgroup have a similar community structure and highlighted a low prevalence of known, highly pathogenic phyla in samples in the False Negatives group as well as in Treated samples.

OTU level analysis highlighted that bacterial species previously associated with aggressive and refractory periodontitis [24] are, indeed, heavily reduced by the Perioblast ${ }^{\mathrm{TM}}$ protocol, and that most of them are poorly represented in cases of the False Negatives group. SIMPER analysis, performed at the OTU level to identify new candidate pathogens among False Negatives, revealed new bacterial species potentially involved in aggressive periodontitis, some of which are abundant in cases where the known pathogens are absent. Notably, some of these are defined as opportunistic pathogens in various extra-oral infections. In particular, Porphyromonas and Prevotella species have a role in the pathogenesis of rheumatoid arthritis [40], while the colonisation of periodontal pockets by Leptotrichia, Cardiobacterium, Porphyromonas, Actinomyces and Aggregatibacter could be a precursor of endocarditis or atheromatous plaques [41], but also intraamniotic infection, neonatal infection (Leptotrichia) [42] or septic arthritis (Actinomyces) [43].

Despite microbiological analyses being considered a potentially important approach for periodontal treatments since 1991 [44], and despite periodontal microbiological tests being available since 2000 , no established guidelines exist to apply microbiological analyses as a routine tool in the management of periodontitis. Evaluation of the clinical results described here should also take into account three important benefits intrinsically associated with laser treatment. While in works describing the outcome of standard treatment SRP is also adopted in follow-up courses, the PERIOBLAST protocol envisions, at follow-up, only maintenance laser therapy, which is substantially less invasive and allows better preservation of dental surfaces. Moreover, the effective and durable pocket decontamination achieved by laser treatment, in addition to destroying the infectivity of all microorganisms, including viruses involved in the onset and progression of periodontitis [45], allows reducing local and systemic antibiotic treatment and, consequently, the risk of driving antibiotic resistance. This is a benefit not only for the patient but also for public health, in particular considering high-risk subjects such as children and elderly people [46].

Finally, thanks again to its microbicidal activity, the efficacy of laser therapy is less dependent on the operator than flapless SRP, whose success relies heavily on the experience and skills of the operator. This is confirmed by the fact that similar results were obtained in all centres applying the laserbased protocol. Considering the above advantages, the Perioblast ${ }^{\mathrm{TM}}$ protocol should be preferred over standard periodontal treatment, even in case of comparable clinical results, to minimise invasiveness and use of antibiotics, while at the same time achieving reproducible efficacy.

\section{Conclusion}

In the field of periodontal disease, the clinical-microbiological study described here is unprecedented in terms of sample numerosity and follow-up length. Our results clearly show 
that periodontal treatment driven by microbiological profiling produces a significant improvement of all clinical parameters and modifies the bacterial communities of periodontal pockets, restoring an enduring healthy oral ecosystem with a long stability, compared with the $3 / 6$ months currently reported as the maximum interval before periodontal pathogens recolonise the pockets [10]. Also, routine use of microbiological assessment in a follow-up program can provide an early alert in case of incoming relapse. According to the results of the present study, a microbiological follow-up should be carried out every $18 / 24$ months, to allow for timely, quick and noninvasive periodontal treatment, even in the most unstable cases. Such cases, reported in the literature as 'not good responders' [10], could be effectively managed before the development of new severe or irreversible tissue damages.

The results of the metagenomics analysis presented here suggest that pathogenic bacteria not previously associated with periodontitis could be involved in early-onset aggressive cases. The observed efficacy of the Periodontal Biological Laser-Assisted Therapy (Perioblast ${ }^{\mathrm{TM}}$ ) protocol also in such cases suggests that the addition of laser treatment to standard SRP could be more effective thanks to its wider bactericidal spectrum. Additional advantages of the laser-based treatment are reduced invasiveness at follow-up and substantial consistency across operators. In conclusion, it has been shown that periodontal treatment by neodymium-doped yttrium aluminium garnet (Nd:YAG) laser irradiation, guided by accurate microbiological patient characterisation, is consistently effective in modifying oral microbiome and eradicating periodontal pathogens, thereby restoring long-term periodontal health.

The findings described in this study are promising and warrant further investigation through prospective randomised clinical trials testing the efficacy of the treatment approach. Moreover, microbiome next-generation sequencing (NGS) analysis of larger sets of patients pre- and post-treatment is looking to be extremely interesting. Such studies should also explore the potential benefits for patients affected by systemic diseases whose correlation with periodontal pathogens is supported by a steadily increasing literature.

Thanks to the deep and lasting periodontal decontamination achieved through laser therapy, the Perioblast ${ }^{\mathrm{TM}}$ protocol allows minimising the use of antibiotics, thereby contributing to prevent the onset of resistances by bacterial strains also involved in systemic diseases. This benefit is particularly relevant in view of the current worldwide public health efforts aimed at optimising the use of antibiotics, according to the World Health Organization (WHO) guidelines [47].

\section{Compliance with ethical standards}

Conflict of interest The authors have declared that no conflict of interest exists.

\section{References}

1. Darveau RP (2010) Periodontitis: a polymicrobial disruption of host homeostasis. Nat Rev Microb 8(7):481-490. doi:10.1038/ nrmicro2337

2. Pihlstrom BL, Michalowicz BS, Johnson NW (2005) Periodontal diseases. Lancet 366:1809-1820

3. Nibali L, Donos N, Henderson B (2009) Periodontal infectogenomics. J Med Microbiol 58:1269-1274. doi:10.1099/ jmm.0.012021-0

4. Quirynen M, Vogels R, Peeters W, van Steenberghe D, Naert I, Haffajee A (2006) Dynamics of initial subgingival colonization of 'pristine' peri-implant pockets. Clin Oral Implants Res 17:25-37

5. Seymour GJ, Ford PJ, Cullinan MP, Leishman S, Yamazaki K (2007) Relationship between periodontal infections and systemic disease. Clin Microbiol Infect 13(Suppl 4):3-10

6. Marakoglu I, Gursoy UK, Marakoglu K, Cakmak H, Ataoglu T (2008) Periodontitis as a risk factor for preterm low birth weight. Yonsei Med J 49(2):200-203. doi:10.3349/ymj.2008.49.2.200

7. Beck JD, Offenbacher S (2001) The association between periodontal diseases and cardiovascular diseases: a state-of-the-science review. Ann Periodontol 6(1):9-15

8. Scannapieco FA, Genco RJ (1999) Association of periodontal infections with atherosclerotic and pulmonary diseases. J Periodontal Res 34(7):340-345

9. Rhemrev GE, Timmerman MF, Veldkamp I, Van Winkelhoff AJ, Van der Velden U (2006) Immediate effect of instrumentation on the subgingival microflora in deep inflamed pockets under strict plaque control. J Clin Periodontol 33(1):42-48

10. Colombo AP, Bennet S, Cotton SL, Goodson JM, Kent R, Haffajee AD, Socransky SS, Hasturk H, Van Dyke TE, Dewhirst FE, Paster BJ (2012) Impact of periodontal therapy on the subgingival microbiota of severe periodontitis: comparison between good responders and individuals with refractory periodontitis using the human oral microbe identification microarray. J Periodontol 83(10):1279-1287

11. Adriaens PA, De Boever JA, Loesche WJ (1988) Bacterial invasion in root cementum and radicular dentin of periodontally diseased teeth in humans. a reservoir of periodontopathic bacteria. J Periodontol 59(4):222-230

12. Johnson JD, Chen R, Lenton PA, Zhang G, Hinrichs JE, Rudney JD (2008) Persistence of extracrevicular bacterial reservoirs after treatment of aggressive periodontitis. J Periodontol 79(12):2305-2312. doi:10.1902/jop.2008.080254

13. Qadri T, Javed F, Poddani P, Tunér J, Gustafsson A (2011) Longterm effects of a single application of a water-cooled pulsed $\mathrm{Nd}$ : YAG laser in supplement to scaling and root planing in patients with periodontal inflammation. Lasers Med Sci 26(6):763-766. doi:10.1007/s10103-010-0807-8

14. Archilla JR, Moreira MS, Miyagi SP, Bombana AC, Gutknecht N, Marques MM (2012) Single session of Nd:YAG laser intracanal irradiation neutralizes endotoxin in dental root dentin. J Biomed Opt 17(11):118002. doi:10.1117/1.JBO.17.11.118002

15. Noguchi T, Sanaoka A, Fukuda M, Suzuki S, Aoki T (2005) Combined effects of Nd:YAG laser irradiation with local antibiotic application into periodontal pockets. J Int Acad Periodontol 7:8-15

16. Aoki A, Mizutani K, Schwarz F, Sculean A, Yukna RA, Takasaki AA, Romanos GE, Taniguchi Y, Sasaki KM, Zeredo JL, Koshy G, Coluzzi DJ, White JM, Abiko Y, Ishikawa I, Izumi Y (2015) Periodontal and peri-implant wound healing following laser therapy. Periodontol 2000 68(1):217-269. doi:10.1111/prd.12080

17. Lei L, Xu J, Reynolds MA, Yan J, Chen Y (2015) High-power Nd: YAG laser triggers the osteogenesis of osteoblasts by activating the bone morphogenetic protein 2 and insulin-like growth factor-1 signaling pathways. Mol Med Rep. doi:10.3892/mmr.2015.3686 
18. Kim IS, Cho TH, Kim K, Weber FE, Hwang SJ (2010) High powerpulsed Nd:YAG laser as a new stimulus to induce BMP-2 expression in MC3T3-E1 osteoblasts. Lasers Surg Med 42(6):510-518. doi:10.1002/lsm.20870

19. Socransky SS, Haffajee AD, Cugini MA, Smith C, Kent RL Jr (1998) Microbial complexes in subgingival plaque. J Clin Periodontol 25(2):134-144

20. Colombo AP, Haffajee AD, Dewhirst FE, Paster BJ, Smith CM, Cugini MA, Socransky SS (1998) Clinical and microbiological features of refractory periodontitis subjects. J Clin Periodontol 25(2):169-180

21. Hughes JB, Hellmann JJ, Ricketts TH, Bohannan BJ (2001) Counting the uncountable: statistical approaches to estimating microbial diversity. Appl Environ Microbiol 67(10):4399-4406

22. Clarke KR (1993) Non-parametric multivariate analyses of changes in community structure. Aust J Ecol 18(1):117-143. doi:10.1111/j. 1442-9993.1993.tb00438.x

23. Ter Braak CJF (1986) Canonical correspondence analysis: a new eigenvector technique for multivariate direct gradient analysis. Ecology 67(5):1167-1179, http://www.jstor.org/stable/1938672, accessed: 27/08/2008

24. Colombo AP, Boches SK, Cotton SL, Goodson JM, Kent R, Haffajee AD, Socransky SS, Hasturk H, Van Dyke TE, Dewhirst F, Paster BJ (2009) Comparisons of subgingival microbial profiles of refractory periodontitis, severe periodontitis, and periodontal health using the human oral microbe identification microarray. J Periodontol 80(9):1421-1432. doi:10.1902/jop.2009.090185

25. Winkel EG, Van Winkelhoff AJ, Timmerman MF, Van der Velden U, Van der Weijden GA (2001) Amoxicillin plus metronidazole in the treatment of adult periodontitis patients. A double-blind placebo-controlled study. J Clin Periodontol 28(4):296-305

26. Garcia Canas P, Khouly I, Sanz J, Loomer PM (2015) Effectiveness of systemic antimicrobial therapy in combination with scaling and root planing in the treatment of periodontitis: a systematic review. J Am Dent Assoc 146(3):150-163

27. Heitz-Mayfield LJA, Trombelli L, Heitz F, Needleman I, Moles D (2002) A systematic review of the effect of surgical debridement vs non-surgical debridement for the treatment of chronic periodontitis. J Clin Periodontol 29(Suppl 3):92-102

28. Adriaens PA, Adriaens LM (2004) Effects of nonsurgical periodontal therapy on hard and soft tissues. Periodontol 2000 36:121-145

29. Bollen CM, Vandekerckhove BN, Papaioannou W, Van Eldere J, Quirynen M (1996) Full- versus partial-mouth disinfection in the treatment of periodontal infections. A pilot study: long-term microbiological observations. J Clin Periodontol 23(10):960-970

30. Colombo AP, Teles RP, Torres MC, Rosalém W, Mendes MC, Souto RM, Uzeda MD (2005) Effects of non-surgical mechanical therapy on the subgingival microbiota of Brazilians with untreated chronic periodontitis: 9-month results. J Periodontol 76(5):778-784

31. Cugini MA, Haffajee AD, Smith C, Kent RL Jr, Socransky SS (2000) The effect of scaling and root planing on the clinical and microbiological parameters of periodontal diseases: 12-month results. J Clin Periodontol 27(1):30-36

32. Feres M, Haffajee AD, Allard K, Som S, Socransky SS (2001) Change in subgingival microbial profiles in adult periodontitis subjects receiving either systemically-administered amoxicillin or metronidazole. J Clin Periodontol 28(7):597-609
33. Haffajee AD, Patel M, Socransky SS (2008) Microbiological changes associated with four different periodontal therapies for the treatment of chronic periodontitis. Oral Microbiol Immunol 23(2):148-157

34. Ioannou I, Dimitriadis N, Papadimitriou K, Sakellari D, Vouros I, Konstantinidis A (2009) Hand instrumentation versus ultrasonic debridement in the treatment of chronic periodontitis: a randomized clinical and microbiological trial. J Clin Periodontol 36(2):132-141

35. Matarazzo F, Figueiredo LC, Cruz SE, Faveri M, Feres M (2008) Clinical and microbiological benefits of systemic metronidazole and amoxicillin in the treatment of smokers with chronic periodontitis: a randomized placebo-controlled study. J Clin Periodontol 35(10):885-896

36. Paolantonio M, D’Angelo M, Grassi RF, Perinetti G, Piccolomini R, Pizzo G, Annunziata M, D’Archivio D, D'Ercole S, Nardi G, Guida L (2008) Clinical and microbiologic effects of subgingival controlled-release delivery of chlorhexidine chip in the treatment of periodontitis: a multicenter study. J Periodontol 79(2):271-282

37. Zijnge V, Meijer HF, Lie MA, Tromp JA, Degener JE, Harmsen HJ, Abbas F (2010) The recolonization hypothesis in a full-mouth or multiple-session treatment protocol: a blinded, randomized clinical trial. J Clin Periodontol 37(6):518-525

38. Heller D, Varela VM, Silva-Senem MX, Torres MC, Feres-Filho EJ, Colombo AP (2011) Impact of systemic antimicrobials combined with anti-infective mechanical debridement on the microbiota of generalized aggressive periodontitis: a 6-month RCT. J Clin Periodontol 38(4):355-364

39. Johnson JD, Chen R, Lenton PA, Zhang G, Hinrichs JE, Rudney JD (2008) Persistence of extracrevicular bacterial reservoirs after treatment of aggressive periodontitis. J Periodontol 79(12):2305-2312

40. Scher JU, Abramson SB (2013) Periodontal disease, Porphyromonas gingivalis, and rheumatoid arthritis: what triggers autoimmunity and clinical disease? Arthritis Res Ther 15(5):122

41. Rath SK, Mukherjee M, Kaushik R, Sen S, Kumar M (2014) Periodontal pathogens in atheromatous plaque. Indian J Pathol Microbiol 57(2):259-264. doi:10.4103/0377-4929.134704

42. Han YW, Shen T, Chung P, Buhimschi IA, Buhimschi CS (2009) Uncultivated bacteria as etiologic agents of intra-amniotic inflammation leading to preterm birth. J Clin Microbiol 47(1):38-47. doi: 10.1128/JCM.01206-08

43. Lequerré T, Nouvellon M, Kraznowska K, Bruno MC, Vittecoq O, Mejjad O, Daragon A, Le Loët X (2002) Septic arthritis due to Actinomyces naeslundii: report of a case. Joint Bone Spine 69(5): 499-501

44. van Winkelhoff AJ, de Graaff J (1991) Microbiology in the management of destructive periodontal disease. J Clin Periodontol 18(6):406-410

45. Martelli FS, Bacci G, Martelli ML, Nobili P, Boddi A, Rosati C, Fanti E (2015) Efficacy of the ND:YAG laser therapy on EBV and HSV1 contamination in periodontal pockets. Ig Sanità Pubbl 71: 369-385

46. Das P, Horton R (2015) Antibiotics: achieving the balance between access and excess. Lancet. pii: S0140-6736(15)00729-1

47. Abdula N, Macharia J, Motsoaledi A, Swaminathan S, VijayRaghavan K (2015) National action for global gains in antimicrobial resistance. Lancet. S0140-6736(15)00668-6 